Letters to the Editor

\section{Contamination of Guidewires During Insertion of Central Venous Catheters in an Intensive Care Setting}

\section{To the Editor:}

Central venous catheters (CVCs) remain a cornerstone of the management of intensive care unit (ICU) patients, allowing measurement of central venous pressure and administration of therapeutic agents. Insertion of a CVC involves puncture of the target vein with a large-bore hollow needle, followed by insertion of a soft J-tipped guidewire through the needle. After dilation, the catheter is advanced into the vein to the desired distance. The guidewire is then removed, the lumens are aspirated and flushed, and the catheter is secured. In theory, the guidewire should remain sterile throughout the procedure.

Use of full sterile gown and gloves, sterile drapes covering a large area, a large sterile area for the equipment required, and an appropriate antiseptic skin preparation would be considered appropriate. ${ }^{1}$

During difficult insertion, multiple manipulations of the equipment occur and the drapes often move, potentially exposing areas of skin that have not been appropriately cleansed.

Infection of CVCs is one of the most important complications of CVC insertion. ${ }^{2}$ The risk factors for and pathogenesis of infection have been well described. ${ }^{3}$ We hypothesized that a poor insertion technique might result in detectable contamination of guidewires.

Royal Prince Alfred Hospital is a 650-bed, tertiary-care referral hospital with general medical and surgical and specialized cardiothoracic and neurosurgical ICUs. Protocols for insertion were identical across the units. Topical antisepsis was achieved with chlorhexidine gluconate and alcohol (BD Persist Plus, Becton Dickinson, Sandy, UT) and Opsite IV 3000 dressings (Smith \& Nephew, Hull, England) were used.
All patients needing new CVCs were recruited from the Intensive Care Services Department between March and December 2002. Subclavian, internal jugular, and femoral vein cannulation were assessed. ArrowHowes catheters (Arrow International, Reading, PA) were used for central venous access and Niagra catheters (Niagra Bard, Ontario, Canada) were used for dialysis. No catheters were antibiotic impregnated or coated, and all guidewires were springwire guide $\mathrm{J}$ tip.

Department staff were educated regarding the method for swabbing guidewires. Importantly, staff were instructed to keep guidewires within the sterile field. All CVC kits in the department had study protocols attached. Demographics were collected for each patient, including operator grade, insertion site, and insertion difficulty. After successful insertion of a CVC, an Interpath Services $\mathrm{P} / \mathrm{C}$ sterile transport swab (Interpath Services, Heidelberg, Victoria, Australia), dampened with sterile saline, was taken from the guidewire prior to removal from the sterile field. The swab was run down the length of the guidewire twice. Swabs were sent immediately to the microbiology department, where they were subjected to routine plating on solid culture media. The existing unit policy to send all catheter tips to the microbiology department after removal was followed. Catheter tips were assessed by the Maki roll technique. Guidewires were considered colonized if any organisms were detected. The protocol was approved by the local ethics committee. Data were assessed by chi-square test with SPSS software (SPSS, Inc., Chicago, IL).

A total of $158 \mathrm{CVC}$ guidewires from 99 patients were studied. Twenty-four were femoral, 83 were internal jugular, and 51 were subclavian. Thirteen guidewires tested positive for microorganisms (8.2\%). Among these, 9 of the CVC tips were analyzed on removal, with 7 (77\%) demonstrating colonization and 4 (31\%) of these having greater than 15 colony-forming units. Among the 145 negative guidewires, 94 CVC tips were assessed on removal. Forty-one (44\%) of these were colonized, with 22 (15\%) having greater than 15 colony-forming units. The organisms found on the contaminated guidewires included coagulase-negative staphylococci in $60 \%$, Escherichia coli in $20 \%$ (both in the same patient at different times), Staphylococcus aureus in $10 \%$, and Corynebacterium species in $10 \%$. Coagulase-negative staphylococci accounted for $66 \%$ of the colonized catheters, Staphylococcus aureus for $32 \%$, and gram-negative organisms for the remainder.

The same organism was recovered from the guidewire and the paired catheter tip in $6(86 \%)$ of the 7 cases. Colonization of the guidewire was predictive of subsequent colonization of the catheter tip $(P=.05)$ and had a positive predictive value of $77 \%$. The sensitivity of guidewire contamination was only $14 \%$, but the specificity was $96 \%$. With the use of 15 colony-forming units as the cutoff for analysis, the same organism was found in 4 of the cases. The duration of catheter insertion was 4.7 days (range, 1 to 9 days) in the group with contamination, compared with 7.6 days (range, 2 to 32 days) in the group with no contamination $(P=.007)$. Overall, $27 \%$ of the catheter tips were colonized when 15 colony-forming units was used as the cutoff.

Difficulty of insertion was selfreported on a scale of 1 to 3 , with 1 being insertion at first or second pass, 2 meaning more than three passes were required, and 3 meaning change of insertion site, operator, or both was required. Contamination of guidewires appeared to increase with insertion difficulty: 6 (7\%) of 88 of grade 1 difficulty, 2 (9\%) of 23 of grade 2 difficulty, and $3(18 \%)$ of 17 of grade 3 difficulty were contaminated.

Contamination of guidewires occurred regardless of insertion site, with 4 of 24 femoral, 4 of 83 internal jugular, and 2 of 51 subclavian guidewires contaminated.

This was designed as a pilot study to determine whether and to what extent guidewire contamination occurs. Guidewire contamination probably occurs due to multiple manipulations of the guidewire, inadequate 
preparation of the sterile field, and poor insertion technique. We believe that, as a quality assurance tool, it may be useful to assess compliance with protocols for catheter insertion. Correlation with accepted definitions of catheter infection and catheter-associated bacteremia was not attempted in this study. Other methods for detecting the small number of organisms on the guidewire that may be more sensitive include immersing it directly into culture medium or direct impression of it onto a solid culture medium. Our method reduces manipulation of the guidewire and thus the risk of postinsertion contamination and is simple to apply. Further study is required to determine whether routine testing of guidewires has any role in predicting early colonization of CVCs.

\section{REFERENCES}

1. Grady NP, Alexander M, Dellinger EP, et al. Guideline for prevention of intravascular catheter-related infections, 2002. MMWR Recomm Rep 2002;51 (RR-10):1-26

2. Raad I. Intravascular catheter-related infections. Lancet 1998;351:893-898.

3. Polderman KH Girbes ARJ. Central venous catheter use: Part 2. Infectious complications. Intensive Care Med 2002;28:18-28.

Susanah Palmer, MBChB, DRCOG Thomas Solano, MBBS, FRACP, FJFICM

Intensive Care Unit Royal Prince Alfred Hospital Camperdown, New South Wales,

Australia

Positive Predictive Value of a Percutaneously Drawn Blood Culture Growing Skin Flora Varies Markedly by Organism

\section{To the Editor:}

Positive blood cultures may be the result of contamination when a patient's skin flora is introduced into the blood specimen during collection. Coagulase-negative staphylococci are the leading cause of bloodstream infections (BSIs) in hospitals, 1,2 but they are also frequent blood culture contaminants. ${ }^{3}$ Judging the clinical meaning of blood cultures positive for common skin organisms is essential but often difficult. The authors of guidelines on the management of catheter-related BSIs and others have recommended requiring multiple blood culture sets

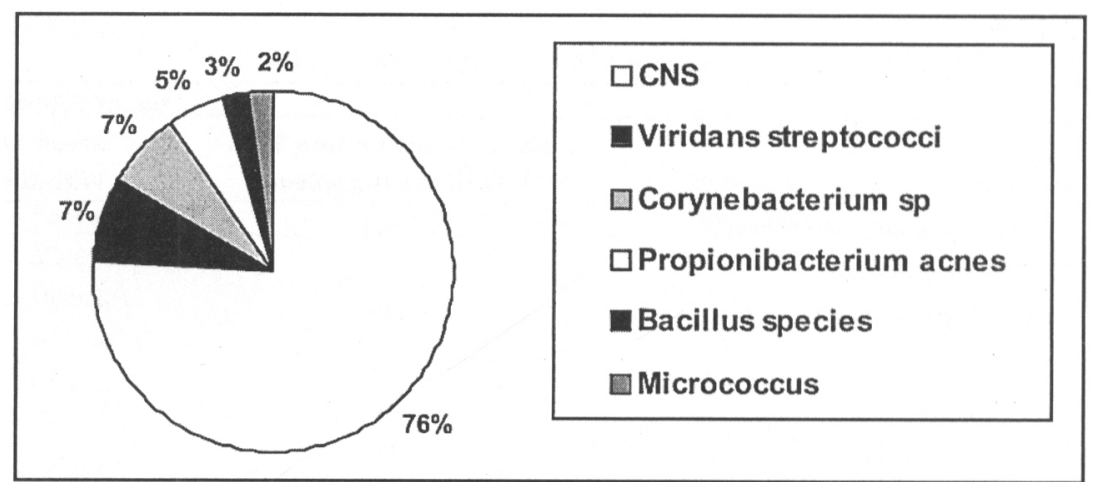

FIGURE. Distribution of species in the blood cultures with skin organisms. CNS = coagulase-negative staphylococci.

positive for the same skin organism. ${ }^{4-6}$ However, sometimes a second blood culture set is unavailable, which can make interpretation of growth of common skin organisms in a single blood culture set difficult. ${ }^{\text {? }}$

This study sought to evaluate blood culture practices at a university hospital, to determine the relative frequency distribution of skin organisms in percutaneously drawn blood cultures, and to calculate the predictive value of a first blood culture positive for common skin organisms in situations where multiple blood cultures were available for interpretation.

This retrospective study was conducted in a 600 -bed hospital. Computerized clinical microbiology laboratory data from October 1998 through September 2003 were used. In this hospital, blood cultures were drawn only from patients with symptoms suggesting BSI, such as fever; clinicians were advised to draw multiple blood culture sets from different sites for evaluating a new fever; and clinicians had been required to reveal whether a blood culture was percutaneous or drawn from a central venous catheter or from an arterial catheter. This labeling had been found to be usually correct in discussing individual cases with attending physicians, but no formal study of the accuracy of clinician labeling of percutaneous blood cultures was conducted.

A blood culture set consisted of a pair of blood culture bottles, usually one aerobic and the other anaerobic; they were usually both inoculated from a single blood specimen drawn at one site. All blood culture sets labeled percutaneous and positive for common skin organisms (ie, coagu- lase-negative staphylococci, Micrococcus species, Propionibacterium acnes, viridans streptococci, Corynebacterium species other than group $\mathrm{JK}$, or Bacillus species) were classified using the following definition: if a common skin organism was isolated from only one of multiple sets of blood cultures (which, in some patients, included one or more catheter-drawn blood cultures) drawn within a 5-hour period, it was considered "contaminated." Each single culture was classified as "probably true" if the same common skin organism was isolated from two or more sets of blood cultures drawn at different times. For this purpose, data such as species and antibiogram from routine clinical microbiology testing were used when available, but the laboratory did not always do such testing. Cultures yielding a common skin organism for which there was no companion set of blood cultures for comparison were excluded from the analysis. The positive predictive value of growing any common skin organism and of growing each type of skin organism was calculated. Positive predictive values of each type of skin organism were compared using chi-square or Fisher's exact test when appropriate.

During the study period, 3,356 (4.9\%) of 69,163 percutaneously drawn blood culture sets were positive for a common skin organism. These 3,356 positive blood culture sets were drawn during 3,139 episodes possibly suggestive of BSI (eg, onset of new fever). Of these episodes, 335 (10.7\%) were considered to represent true bacteremia due to a common skin organism, with 213 episodes being confirmed by additional percutaneous blood culture sets, 110 by blood cul- 